\title{
OPTICAL PROPERTIES OF DILUTED MAGNETIC SEMICONDUCTOR QUANTUM STRUCTURES
}

\author{
M. Dobrowolska, H. Luo and J.K. Furdyna \\ Department of Physics, University of Notre Dame, Notre Dame, IN 46556, USA
}

\begin{abstract}
Conduction and valence band edges in diluted magnetic semiconductors undergo enormous Zeeman shifts when a magnetic field is applied, reaching values in excess of $100 \mathrm{meV}$ at low temperatures. These Zeeman shifts can thus have profound consequences on the properties of DMS/non-DMS heterostructures, since they provide the opportunity of tuning their band alignment by varying an applied field. This leads to a variety of entirely new effects, and also provides a powerful tool for probing the effect of band alignment on the properties of semiconductor heterostructures in general. We illustrate this with several examples. First, using the $\mathrm{ZnSe} / \mathrm{ZnMnSe}$ system, we discuss the creation of a spatial spin modulation (spin superlattice). Second, we use the drastic differences in the Zeeman splitting occurring in different layers of a DMS/non-DMS superlattice in order to pinpoint the localization in space of the specific electronic states involved in optical transitions. We illustrate this by investigating the localization of above-barrier states in type-I $\mathrm{ZnSe} / \mathrm{ZnMnSe}$ superlattices, and of spatially-direct (type-I) excitons which occur in $\mathrm{ZnTe} / \mathrm{CdMnSe}$ and $\mathrm{ZnMnTe} / \mathrm{CdSe}$ type-II superlattices. Finally, we exploit Zeeman tuning to demonstrate the confinement effects which occur in a single quantum barrier.
\end{abstract}

PACS numbers: 73.20.Dx, 75.30.Et, 78.20.Ls, 68.55.Bd

\section{Introduction}

Diluted magnetic semiconductors (DMSs) are semiconducting ternary or quaternary alloys whose lattice consists in part of substitutional magnetic ions $[1,2]$. $\mathrm{Zn}_{1-x} \mathrm{Mn}_{x} \mathrm{Se}, \mathrm{Cd}_{1-x} \mathrm{Mn}_{x} \mathrm{Te}$, or $\mathrm{Pb}_{1-x} \mathrm{Mn}_{x} \mathrm{Te}$ are examples of such systems. One of the most interesting and important properties of DMSs is the spin-spin exchange interaction between the magnetic ions in the lattice and the band electrons. When an external magnetic field is applied, this interaction - referred to as the $s p-d$ interaction - leads to an enormous amplification of Zeeman splittings of electronic levels (band edges, impurity levels, etc.), which at low temperatures can reach values in excess of $100 \mathrm{meV}$ in moderate magnetic field (e.g., 5 tesla). The origin of such $s p-d$ amplification of the Zeeman splitting has been extensively discussed in 


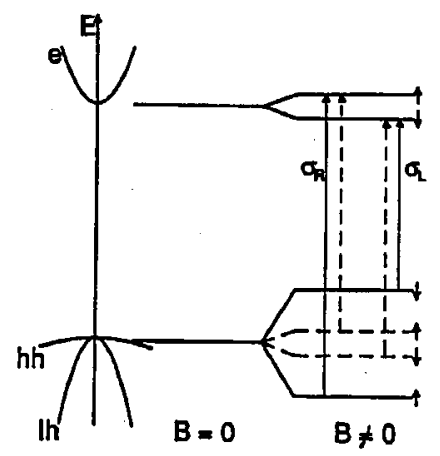

Fig. 1. A schematic diagram of Zeeman splittings of the conduction and valence band edges. Spin-up and spin-down states are symbolized by arrows, and the light-hole band splitting is shown by horizontal dashed lines. Allowed interband transitions are shown by vertical lines, with corresponding circular polarizations $\left(\sigma_{\mathrm{L}}\right.$ and $\left.\sigma_{\mathrm{R}}\right)$ indicated.

the literature $[1,2]$. Here we simply note that the amount of splitting is different for different bands, and that in wide-gap DMSs the Zeeman splitting far exceeds the Landau splitting, so that the latter is usually neglected.

These features are conveniently illustrated by Fig. 1. Note, in particular, that the splitting of the heavy-hole band is considerably larger than for the conduction band, and is opposite in sign. Therefore the spin-conserving transitions (indicated by arrows in Fig. 1) will be strongly red shifted for one sense of circular polarization and blue shifted for opposite. Since optical transitions involving light holes (broken lines in Fig. 1) are generally much weaker than those associated with heavy holes, in this brief presentation we will restrict ourselves to effects associated with heavy holes only.

The $s p-d$ exchange interaction has particularly exciting implications for quantum wells and superlattices consisting of non-magnetic and DMS layers, where it provides a handle for "tuning" the relative alignment of the band edges over a significant scale (typically over several tens of meV) simply by varying an applied magnetic field. This tuning leads to new effects, and also provides a tool for studying general properties of semiconductor heterostructures such as mapping the wave function distribution in real space and investigating subbands formation in superlattices with very small band offsets.

\section{Spin superlattices}

A "spin superlattice" (SSL) is a superlattice in which carriers with opposite spin states are confined in different layers. In order to achieve such spin-modulation, we look for a structure in which the energy gaps of the constituent layers are initially (i.e., in the absence of magnetic field) equal, and the band offsets at the interfaces are initially zero, as shown at the top of Fig. 2 (where DMS regions are designated by shading). When a magnetic field is applied, the large Zeeman splitting of the band edges in the DMS layers results in induced band offsets and, 

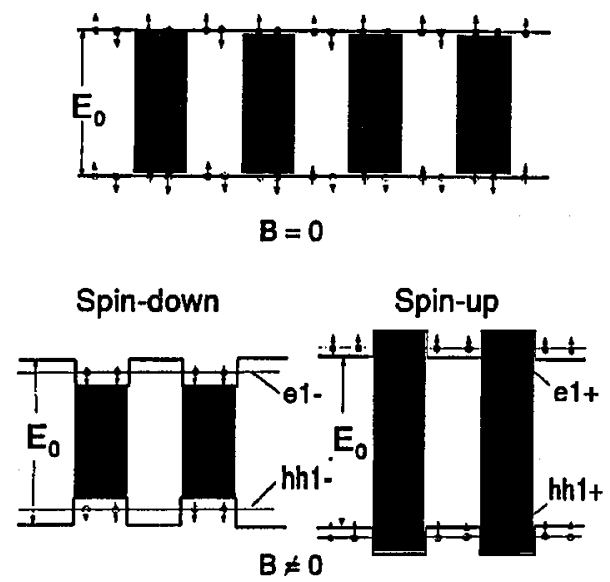

Fig. 2. A schematic diagram of the band structure of a magnetic-field-induced spin superlattice, consisting of DMS (shaded) and non-DMS (unshaded) layers with equal energy gaps at $B=0$ (upper panel). Lower panel shows the diagram at $B \neq 0$ for carriers with spin-down (left) and spin-up (right) orientation.

consequently, in a spatial separation of the spin-up and spin-down states, as shown in Fig. 2 (bottom). The SSL phenomenon has already been observed experimentally in several DMS/non-DMS multi-quantum well systems [3, 4].

One particularly attractive DMS system for achieving the structure described above is the $\mathrm{ZnSe} / \mathrm{Zn}_{1-x} \mathrm{Mn}_{x}$ Se superlattice. The energy gap of $\mathrm{Zn}_{1-x} \mathrm{Mn}_{x} \mathrm{Se}$ exhibits a rather striking bowing with Mn concentration $x$ at low temperatures, first decreasing with $x$, and then rapidly increasing [5]. It is thus possible to find a value of $x$ at which $\mathrm{Zn}_{1-x} \mathrm{Mn}_{x}$ Se has the same energy gap as $\mathrm{ZnSe}$. This occurs for $x \approx 0.04$ at $1.5 \mathrm{~K}$. It turns out that this value of $x$ also leads to zero (or, in practice, to very small) band offsets in both the conduction and the valence bands, thus satisfying the properties stipulated in the preceding paragraph.

Examining Fig. 1 we note that, when the magnetic field is applied, the band edge of $\mathrm{Zn}_{1-x} \mathrm{Mn}_{x}$ Se for spin-down electrons will move down, and it will move up in energy for spin-down holes. The $\mathrm{Zn}_{1-x} \mathrm{Mn}_{x}$ Se layers thus become wells for both carriers of the spin-down orientation. Similarly, for spin-up states the conduction band moves up in energy, and the heavy-hole band moves down, so that for this spin orientation the $\mathrm{ZnSe}$ layers become the wells. This magnetic-field-induced band offset has therefore the consequence of spatially separating electron and hole states of different spin orientation. Such spin separation can be readily observed in magnetoabsorption experiments in the Faraday geometry, in which only spin-conserving transitions are allowed $(\Delta S=0)$. Using left- and right-handed circular polarizations (from here on designated by $\sigma_{L}$ and $\sigma_{R}$ ), one can then observe separately transitions between spin-up valence and conduction band states (corresponding to $\left.\sigma_{\mathrm{R}}\right)$, and those between spin-down states $\left(\sigma_{L}\right)$. 


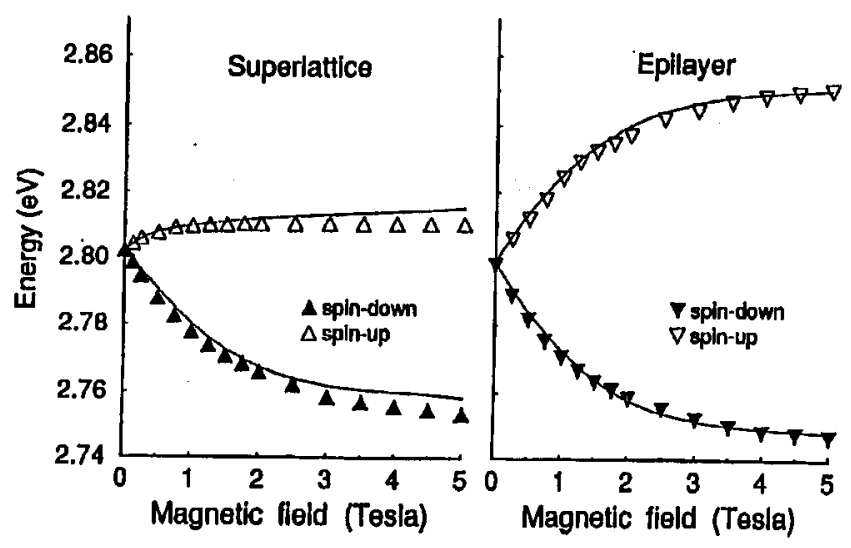

Fig. 3. Experimental energies of the Zeeman-split excitonic ground state transitions in a $\mathrm{Zn}_{.96} \mathrm{Mn}_{.04} \mathrm{Se} / \mathrm{ZnSe}(100 \AA / 100 \AA)$ SSL for the two spin orientations (left). Note the striking asymmetry in the Zeeman shift for the spin-up and spin-down transitions relative to $B=0$, as compared to the Zeeman shift observed for a "companion" epilayer of $\mathrm{Zn}_{.96} \mathrm{Mn}_{0.04} \mathrm{Se}$ (right). Lines are the results of calculation.

This is illustrated in Fig. 3 (left), which shows the magnetic field dependence of excitonic transitions observed with the $\sigma_{\mathrm{L}}$ and $\sigma_{\mathrm{R}}$ polarization in a $\mathrm{ZnSe} / \mathrm{Zn}_{.96} \mathrm{Mn}{ }_{.04} \mathrm{Se}$ SSL. Considering first the spin-down $\left(\sigma_{\mathrm{L}}\right)$ transitions, we see the rapid red shift of the $\sigma_{\mathrm{L}}$ absorption line, since the initial and final states of the transition are both in the DMS layers, and thus follow the magnetic field dependence of the valence and conduction band edges of the DMS material (see Fig. 1). In contrast, the spin-up transitions take place between states localized in the $\mathrm{ZnSe}$ layers, which become the wells for this spin orientation when the field is applied. This is responsible for the flatness of the transition energy at higher fields, i.e., once the offset becomes well defined. The small initial rise in energy seen in Fig. 3 at low fields (i.e., when the offset is just beginning to form) arises from the fact that in that region of extremely shallow wells the state in the $\mathrm{ZnSe}$ well is much more sensitive to the height of the (DMS) barrier, which increases with the field. As the barrier continues to increase, the energy of the state confined in the $\mathrm{ZnSe}$ well becomes less and less sensitive to the barrier height, eventually approaching the flat behavior expected for states confined in non-magnetic quantum wells $[3,4]$.

For comparison, we show on the right of Fig. 3 the position of free exciton magnetoabsorption data observed for $\sigma_{\mathrm{L}}$ and $\sigma_{\mathrm{R}}$ polarizations in an epilayer grown with the same $\mathrm{Mn}$ concentration as the $\mathrm{Zn}_{1-x} \mathrm{Mn}_{x}$ Se layers of the SSL. Note that, in contrast with the SSL data, the $\sigma_{\mathrm{L}}$ and $\sigma_{\mathrm{R}}$ branches for the epilayer are quite symmetric. The asymmetry seen on the left-hand side is a characteristic signature of spin-separating structures.

\section{Mapping of wave functions in heterostructures}

As already pointed out, heterostructures consisting of alternating DMS and non-DMS layers provide a unique opportunity for studying wave function (or 
probability) distributions. The Zeeman splitting of a given state will depend on how the wave function of that state is distributed between the DMS and the non-DMS layers: subbands strongly localized in DMS layers will exhibit large splittings, while Zeeman splittings of states localized in non-DMS layers will be weak.

While localization of carriers is fairly obvious for low-lying states in deep quantum wells, this issue is not at all clear for subbands at energies above the top of the barriers. Unit recently little was known about such above-barrier subbands, the first observation of optical transitions involving these states having been made only a few years ago [6-8]. Since then it has become clear that such high-lying subbands participate strongly in optical transitions due to the formation of localized states in the barrier region [9-13]. Localization at energies above the top of the barrier originates from constructive interference of the wave functions reflected at barrier-well interfaces [14].

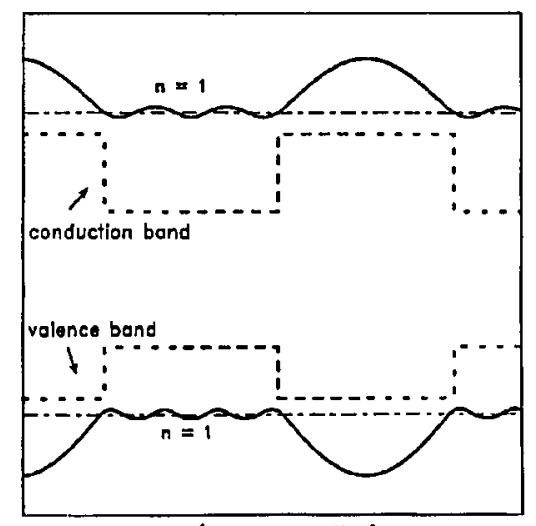

$z$ (growth direction)

Fig. 4. The band structure of a type-I superlattice (solid lines) and the wave functions (dashed lines) of the first above-barrier subbands for both the conduction and the valence bands. The eigenenergies at the center of the superlattice Brillouin zone which correspond to the wave functions shown are plotted by the dash-dotted lines. The localization of the subbands results in the formation of above-barrier excitons localized in the barrier regions of the superlattice.

For large band offsets, and for low-lying above-barrier states, a clear relationship can be established between the shape of the wave function and a new quantum number, obtained by counting not from the bottom of the well, but from the top of the barriers [14]. For example, when the first above-barrier subband lies close to the energy corresponding to $K_{\mathrm{b}}=\pi / L_{\mathrm{b}}$ (where $K_{\mathrm{b}}$ is the wave vector in the barrier region, and $L_{\mathrm{b}}$ is the barrier layer thickness), the wave function for states in this subband will have the shape in the barriers similar to that of a ground state of the superlattice in the wells (see Fig. 4). In this section we will discuss the localization of above-barrier states in DMS/non-DMS type-I and type-II superlattices and also of quasi-bound states in a single quantum barrier. 


\section{1. $Z n C d S e / Z n M n S e$ type-I superlattices}

Figure 4 shows the wave functions of the first above-barrier subbands in the conduction and the valence bands for a type-I superlattice. Such localized above-barrier electrons and holes can then form excitons (which will also be localized in the barriers). This has been clearly demonstrated experimentally for type-I $\mathrm{ZnCdSe} / \mathrm{ZnMnSe}$ superlattices [9]. The band structure of such superlattices is depicted in the inset of Fig. 5. Since the energy gap of $\mathrm{ZnCdSe}$ is considerably smaller than that of $\mathrm{ZnMnSe}$ for the $\mathrm{Cd}$ and $\mathrm{Mn}$ concentrations used here, the wells in this system correspond to the nonmagnetic layer, while the barriers are magnetic.

Figure 5 shows a low-temperature $(1.5 \mathrm{~K})$ absorption spectrum in the absence of an external magnetic field for $\mathrm{Zn}_{0.88} \mathrm{Cd}_{0.12} \mathrm{Se} / \mathrm{Zn}_{0.90} \mathrm{Mn}_{0.10} \mathrm{Se}$, with the well and barrier widths both equal to $75 \AA$. Several excitonic absorption peaks are observed.

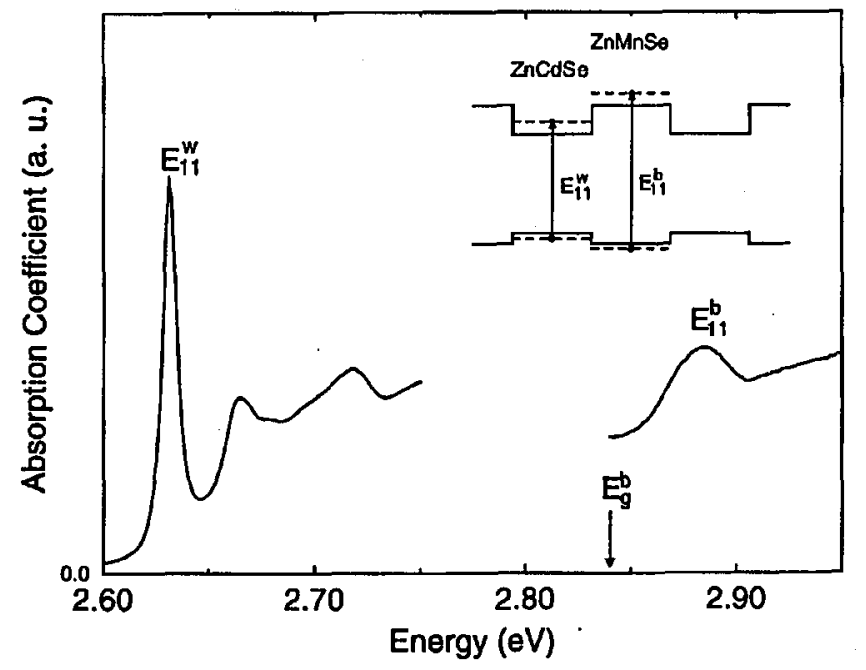

Fig. 5. Absorption spectrum of a $\mathrm{Zn}_{0.88} \mathrm{Cd}_{0.12} \mathrm{Se} / \mathrm{Zn}_{0.90} \mathrm{Mn}_{0.10}$ Se superlat tice observed at $1.5 \mathrm{~K}$ in the absence of a magnetic field. The peak labeled $E_{11}^{w}$ is the excitonic transition associated with the lowest heavy-hole and conduction electron subbands confined in the wells. Transition $E_{11}^{\mathrm{b}}$ is associated with the lowest above-barrier heavy-hole and conduction electron subbands. The arrow labeled $E_{\mathrm{g}}^{\mathrm{b}}$ indicates the energy gap of the barrier material, measured on a $\mathrm{Zn}_{0.9} \mathrm{Mn}_{0.1} \mathrm{Se}$ "companion" epilayer. Inset: band alignment for the $\mathrm{ZnCdSe} / \mathrm{ZnMnSe}$ superlattice.

The peak at $2.631 \mathrm{eV}$ (labeled $E_{11}^{\mathrm{w}}$ ) was identified as the free exciton transition between the first heavy-hole ground state and the conduction electron ground state of the superlattice. The arrow marked $E_{\mathrm{g}}^{\mathrm{b}}$ in the figure indicates the band edge of the barriers, as measured on an epitaxial film of $\mathrm{Zn}_{0.9} \mathrm{Mn}_{0.1}$ Se grown under exactly the same conditions as those used for the barriers. The peak labeled $E_{11}^{\mathrm{b}}$ at $2.886 \mathrm{eV}$ (which is larger than $E_{\mathrm{g}}^{\mathrm{b}}$ ) is identified as the excitonic transition between the first above-barrier heavy-hole state and the first above-barrier electron state 
(the lowest-energy above-barrier transition). In the following we will focus on $E_{11}^{\mathrm{b}}$ and $E_{11}^{\mathrm{w}}$ peaks, and we will not concern ourselves with the other peaks in Fig. 5.

Magnetotransmission data obtained on the $\mathrm{ZnCdSe} / \mathrm{ZnMnSe}$ superlattices have been used to determine the region and degree of spatial localization of wave functions of the states involved in the $E_{11}^{\mathrm{w}}$ and $E_{11}^{\mathrm{b}}$ transitions, as described below. When an external magnetic field is applied, the band edges of the $\mathrm{ZnMnSe}$ barriers will be Zeeman-split, leading in turn to the splitting of optical transitions in the superlattice. Figure 6 shows the energies of the $E_{11}^{\mathrm{w}}$ and $E_{11}^{\mathrm{b}}$ transitions as a

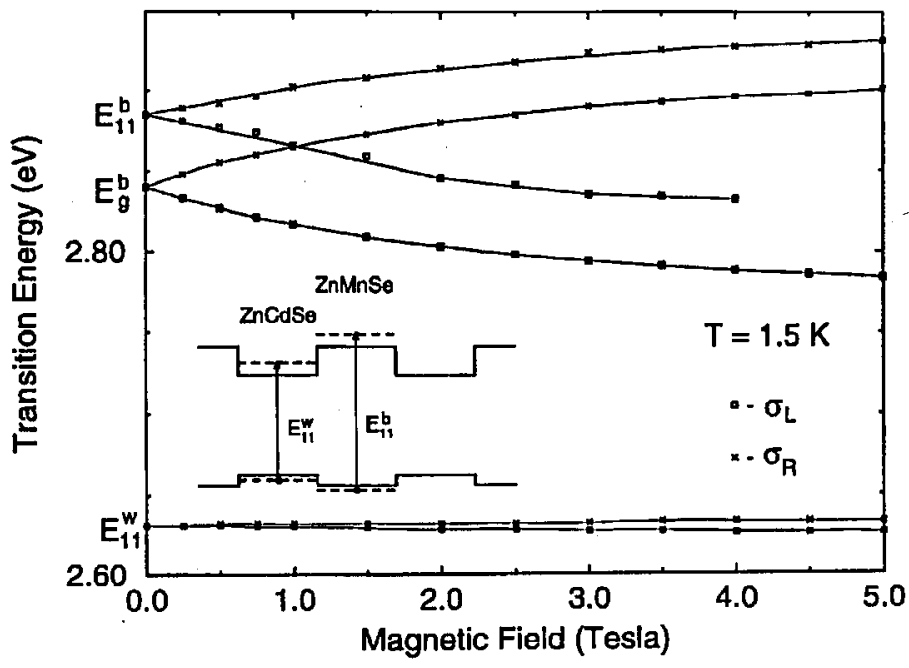

Fig. 6. Spin splittings of the excitonic transitions $E_{11}^{\mathrm{w}}$ and $E_{11}^{\mathrm{b}}$ and of the $E_{\mathrm{g}}^{\mathrm{b}}$ transition obtained from a $\mathrm{Zn}_{0.9} \mathrm{Mn}_{0.1}$ Se epilayer, as a function of magnetic field. The crosses are obtained with $\sigma_{R}$ circular polarizations and represent transitions between spin-up states; the squares correspond to $\sigma_{\mathrm{L}}$ polarizations and represent transitions between spin-down states. The solid lines are guides for the eye.

function of an applied magnetic field. Also shown in the figure is the splitting of the exciton line observed in the epilayer of $\mathrm{Zn}_{0.9} \mathrm{Mn}_{0.1}$ Se having the same $\mathrm{Mn}$ concentration as the barriers of the superlattice, and already used to determine $E_{\mathrm{g}}^{\mathrm{b}}$ in Fig. 5. It is significant that the spin splitting of the above-barrier transition $E_{11}^{\mathrm{b}}$ is clearly much larger than that of the below-barrier transition $E_{11}^{\mathrm{w}}$, and almost (but not quite) as large as that in the $\mathrm{ZnMnSe}$ epilayer.

We recall that the structures investigated are type-I superlattices, consisting of nonmagnetic wells and magnetic barriers. The relatively small (but observable) Zeeman splitting of the ground-state exciton transition $E_{11}^{w}$ (which originates and terminates in the nonmagnetic wells) thus arises from the partial penetration of the wave functions of the initial and final states into the magnetic barriers. By contrast, the much larger Zeeman splitting of $E_{11}^{\mathrm{b}}$ (almost the same as that for the "bulk" $\mathrm{Zn}_{0.9} \mathrm{Mn}_{0.1}$ Se material) indicates that the $E_{11}^{\mathrm{b}}$ transition originates and terminates on states localized predominantly in the DMS (i.e., the barrier) region. 
The fact that the splitting of $E_{11}^{\mathrm{b}}$ is slightly (about $15 \%$ ) below that observed for the $\mathrm{Zn}_{0.9} \mathrm{Mn}_{0.1}$ Se epilayer indicates that a small part of the wave function extends into the non-DMS layers. The data shown in Fig. 6 thus provide direct evidence that the above-barrier excitons in a type-I superlattice are localized in the barrier layers.

\subsection{CdSe/ZnMnTe and CdMnSe/ZnTe type-II superlattices}

Using the same techniques, it was shown that there exist spatially direct type-I-like excitons in type-II superlattices $[10,11]$. The inset in Fig. 7 shows a schematic diagram of the type-II band alignment for a CdMnSe/ZnTe superlattice [11]. In this structure condition electrons at energies below the barriers are localized in the CdMnSe layer, while the below-barrier heavy holes are in the $\mathrm{ZnTe}$ layers. Excitonic transitions have been considered unimportant in such a system, because wave functions of the electrons and the holes are spatially separated, resulting in weak Coulomb attraction.

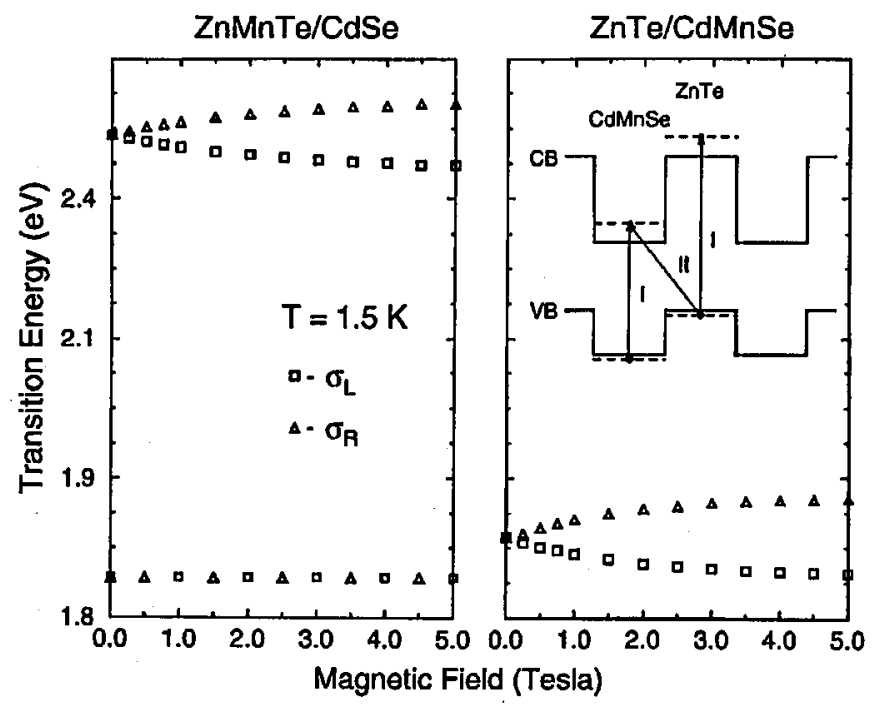

Fig. 7. Spin splittings of the transitions occurring in CdSe layers, and those occurring in $\mathrm{ZnMnTe}$ layers of a CdSe/ZnMnTe superlattice (left); and transitions between states confined in CdMnSe layers in a CdMnSe/ZnTe superlattice (right). Transitions occurring in the $\mathrm{ZnTe}$ layers in the latter superlattice are obscured by the absorption from the $\mathrm{ZnTe}$ buffer layer in this structure. Squares and triangles correspond to transitions between spin-down and spin-up states, respectively. Inset: band alignment and possible transitions in the CdMnSe/ZnTe type-II structure.

As seen in the inset, two types of excitonic transitions are possible in the system illustrated. One takes place between electron states localized in CdMnSe 
wells and hole states in ZnTe wells, as shown by the arrow marked "II" in Fig. 7. This is the well-studied type-II excitonic transition. The other occurs between electron (or hole) subbands confined in the wells, and hole (or electron) subbands at above-barrier energies, confined in the barriers. In that case both the electron and the hole states involved in the transition are localized in the same layer, as shown by the arrows marked "I" in Fig. 7. We shall refer to these spatially direct processes as type-I excitonic transitions.

To show the existence of type-I excitons in type-II superlattices, and to pinpoint their localization, we studied two systems: $\mathrm{CdSe} / \mathrm{ZnMnTe}$, where electrons are localized in a nonmagnetic well; and $\mathrm{CdMnSe} / \mathrm{ZnTe}$, where electrons are localized in a magnetic well. In both cases the type-I excitons confined in the nonmagnetic layer will exhibit very different magneto-optical properties from those confined in the DMS layer. The absorption spectrum measured on $\mathrm{CdSe} / \mathrm{ZnMnTe}$ superlattices revealed two clear excitonic absorption peaks, shown on the left in Fig. 7. The lower energy peak was identified as the type-I (spatially direct) excitonic transition between the lowest electron state confined in the CdSe conduction-band well and the lowest above-barrier hole state confined in the CdSe valence-band barrier layer. The higher-energy peak was attributed to a similar type-I transition, between the above-barrier electron state confined in the $\mathrm{ZnM}$ nTe barrier and the hole state confined in the ZnMnTe well.

The difference in the magnetooptical behavior of both transitions is shown in Fig. 7 , where energies observed for $\sigma_{\mathrm{L}}$ and $\sigma_{\mathrm{R}}$ circular polarizations are plotted as a function of magnetic field. It is quite striking that there is no observable splitting for the lower energy transition, confirming that this transition indeed takes place between states strongly localized in the nonmagnetic CdSe layers. In contrast, there is a large Zeeman splitting for the higher-energy transition, which clearly indicates that this transition occurs between states localized in DMS layers. As before, quantitative information on the degree of localization of the wave function can be extracted by comparing the observed splitting with that exhibited by a $\mathrm{ZnMn}$ Te epilayer grown under exactly the same conditions as the DMS layers of the superlattice.

On the right of Fig. 7 we also show the Zeeman splitting of the lowest-energy absorption peak observed in a $\mathrm{ZnTe} / \mathrm{CdMnSe}$ superlattice. This absorption line exhibits a large Zeeman splitting, indicating that the transition occurs between electrons localized in CdMnSe wells and above-barrier holes localized in the same layers. Both panels of Fig. 7 thus clearly establish the existence of type-I excitons in type-II superlattices.

\subsection{Confinement effects in a single quantum barrier}

The single-barrier system is qualitatively different from most of the thoroughly studied semiconductor heterostructures in that its eigenstates are in the form of a continuum, with no truly bound states. However, when the wavelength of an electron $\lambda$ equals $2 L_{\mathrm{b}} / N$ (where $L_{\mathrm{b}}$ is the barrier width and $N$ is an integer), quasi-localization of the states occurs in the barrier, through interference of traveling electron waves. Such conditions are in fact the same as those for resonant 
tunneling through a single barrier (i.e., the wave functions of states responsible for resonant tunneling through a barrier are quasi-localized in the barrier region). These quasi-bound states will therefore manifest a behavior similar to bound states in quantum wells, such as the dependence of their energy on the band offsets and the layer width. It is particularly striking that these similarities to truly bound states are readily observable in the form of absorption peaks in optical experiments on single barriers - despite the fact that states in this structure do not form discrete levels, or even subbands, but rather a continuum of eigenstates [15].

Although a detailed discussion of the physics of the single barrier is outside the intended scope of this article, it should be clear that the key issue to its behavior is the nature of wave function localization which characterizes this structure. By using a DMS barrier sandwiched in a non-DMS material, such wave function localization can be mapped out in a manner similar to that already described in connection with DMS/non-DMS superlattices. Indeed, this approach has been recently successfully employed using a single $\mathrm{ZnMnSe}$ barrier between ZnSe layers [15], clearly indicating that in this structure both electrons and holes are indeed localized in the barrier region.

\section{Superlattices with small magnetically-induced band offsets}

Small offset DMS/non-DMS superlattices provide a unique laboratory for studying the mini bands formation when the structure is transformed from bulk-like behavior to that of a "regular" superlattice. For superlattices with very small band offsets, a number of new effects are to be expected. First, the smaller offsets do not provide sufficiently strong reflections needed to confine the above-barrier states within the barrier layers (see discussion at the beginning of Sec. 3). This has a profound effect on localization characteristics of above-barrier states, and strongly affects the selection rules which characterize this intermediate dimensionality regime. Furthermore, the small band offsets lead to small mini-band gaps and large subbands widths. Under those conditions it is possible to distinguish transitions taking place at the center of the Brillouin zone from those at the edge of the zone, and thus to measure the widths of the above-barrier minibands. Finally, owing to the width of the subbands, under certain conditions one part of the Brillouin zone may be below the top of the barrier, and the remaining part above, leading to drastically different selection rules for different states even within the same subband (e.g., for the zone center and the zone edge).

An ideal structure for these studies is the $\mathrm{ZnSe} / \mathrm{Zn}_{.96} \mathrm{Mn}_{.04} \mathrm{Se}$ spin superlattice discussed in Sec. 2 because, for all practical purposes, the value of the offsets is determined by the experimenter during the experiment. Dai et al. [16] performed a detailed study of the optical transitions (including those involving above-barrier subbands) on this system, where the band offsets could be continuously tuned over a range from 0 to $40 \mathrm{meV}$ in the valence band, and from 0 to $10 \mathrm{meV}$ in the conduction band as the magnetic field was varied from 0 to $5 \mathrm{~T}$. It is remarkable that in this small-offset region (where the offset does not exceed $0.5 \%$ of the total band gap), evidence was found of subband quantization above the barrier for quantum numbers as high as $n=3$. The ability to accomplish this is made possible by the capability of tuning the offsets as the experiment is being performed. 


\section{Concluding remarks}

We have described several situations involving DMS/non-DMS heterostructures, in which the band alignment in adjacent layers can be continuously tuned by an external magnetic field. The phenomena presented here are intended only as illustrative examples, and the territory covered in this paper is certainly not meant to be exhaustive. Other important applications of Zeeman tuning include the difficult issue of quantitative band offset determination in quantum wells [17], measurements of spin lifetime [18], Zeeman-tunable coupling in double quantum wells separated by DMS barriers [19] and the yet unexplored territory of band-edge tuning relative to impurity levels in modulation-doped structures (magnetic freeze-out and boil-off) $[20,21]$. Undoubtedly these opportunities will increase with further progress in our understanding of the Zeeman processes in heterostructures and especially with further advances in the preparation of new DMS/non-DMS heterostructure configurations.

\section{Acknowledgments}

The authors acknowledge the support of NSF Grant DMR-9208400.

\section{References}

[1] Diluted Magnetic Semiconductors, Eds. J.K. Furdyna, J. Kossut, in series Semiconductors and Semimetals, Vol. 25, Academic Press, Boston 1988.

[2] J.K. Furdyna, J. Appl. Phys. 64, R29 (1988).

[3] W.C. Chou, A. Petrou, J. Warnock, B.T. Jonker, Phys. Rev. Lett. 67, 3820 (1991).

[4] N. Dai, H. Luo, F.C. Zhang, N. Samarth, M. Dobrowolska, J.K. Furdyna, Phys. Rev. Lett. 67, 3824 (1991).

[5] A. Twardowski, T. Dietl, M. Demianiuk, Solid State Commun. 48, 845 (1983).

[6] J.E. Zucker, A. Pinchuk, D.S. Chemla, A. Gossard, W. Weigman, Phys. Rev. B 29, 7065 (1984).

[7] J.J. Song, Y.S. Yoon, A. Fedotowsky, Y.B. Kim, J.N. Schulman, C.W. Tu, D. Huang, H. Morkoc, Phys. Rev. B 34, 8958 (1986).

[8] J.J. Song, Y.S. Yoon, P.S. Jung, A. Fedotowsky, J.N. Schulman, C.W. Tu, J.M. Brown, D. Huang, H. Morkoc, Appl. Phys. Lett. 50, 1269 (1987).

[9] F.C. Zhang, N. Dai, H. Luo, N. Samarth, M. Dobrowolska, J.K. Furdyna, L.R. Ram-Mohan, Phys. Rev. Lett. 68, 3220 (1992).

[10] F.C. Zhang, H. Luo, N. Dai, N. Samarth, M. Dobrowolska, J.K. Furdyna, Phys. Rev. B 47, 3806 (1993).

[11] H. Luo, W.C. Chou, N. Samarth, A. Petrou, J.K. Furdyna, Solid State Commun. 85, 691 (1993).

[12] Lok C. Lew Yan Voon, L.R. Ram-Mohan, H. Luo, J.K. Furdyna, Phys. Rev. B 47, 6585 (1993).

[13] H. Luo, L.R. Ram-Mohan, Y. Xuan, G.L. Yang, J.K. Furdyna, J. Electron. Mater. 22, 1103 (1993).

[14] H. Luo, J.K. Furdyna, Modern Phys. Lett. B 7, 299 (1993). 
[15] H. Luo, N. Dai, F.C. Zhang, N. Samarth, M. Dobrowolska, J.K. Furdyna, C. Parks, A.K. Ramdas, Phys. Rev. Lett 70, 1307 (1993).

[16] N. Dai, L.R. Ram-Mohan, H. Luo, G.L. Yang, F.C. Zhang, M. Dobrowolska, J.K. Furdyna, to be published in Phys. Rev. B.

[17] W.J. Walecki, A.V. Nurmikko, N. Samarth, H. Luo, J.K. Furdyna, N. Otsuka, Appl. Phys. Lett. 57, 466 (1990).

[18] B.T. Jonker, L.P. Fu, W.Y. Yu, W.C. Chou, A. Petrou, J. Warnock, J. Electronic Mater. 22, 489 (1993).

[19] J.F. Smyth, D.D. Awschalom, N. Samarth, H. Luo, J.K. Furdyna, Phys. Rev. B 46 (Rapid Comm.), 4340 (1992).

[20] S. Datta, J.K. Furdyna, R.L. Gunshor, Superlattices Microstruct. 1, 327 (1985).

[21] J. Kossut, J.K. Furdyna, Acta Phys. Pol. A 73, 851 (1988). 